\title{
Grußwort der Präsidentin des Bundesgerichtshofs
}

\section{Von Erfüllungswahl, der Schlacht um die Bastille und einem Heidetraum}

Wenn Vorsitzender Richter am Bundesgerichtshof Professor Dr. Godehard Kayser am 6. Oktober dieses Jabres seinen 65. Geburtstag feiern darf und im Juni des kommenden Jabres in den Rubestand treten muss, dann bat sich ein über 40jähriges Berufsleben im Dienste am Recht erfüllt, das in beeindruckender Weise richterliche Tugenden, wissenschaftliches Profil und persönliche Stärken vereinen konnte. Neben den auch im vorliegenden Werk ersichtlichen insolvenzrechtlichen Schwerpunkten sind auch ein überaus erfolgreiches Wirken für das anwaltliche Berufsrecht sowie die Persönlichkeit eines Richterkollegen zu würdigen, die durch humorvolle, leise Töne ebenso geprägt ist wie durch die Fäbigkeit zu Führung und Gestaltung.

Die deutschen Rechtswissenschaften zeichnen sich in besonderer Weise durch einen offenen und auf hohem Niveau ausgetragenen Diskurs der verschiedenen an ihr beteiligten Professionen aus. Gerade akademische Wissenschaft und richterliches Wirken sind in vielfacher Weise auf diesen sich gegenseitig befruchtenden und auf der Basis derselben Ausbildung gelingenden Austausch angewiesen. Die außerordentlich erfolgreiche Synthese von akademischem und richterlichem Wirken erweist sich als Garant für in besonderer Weise belastbare, nachhaltige und erfolgreiche Rechtanwendung gerade auch bei sich immer wieder stellenden neuen Rechtsfragen, seien sie durch den Gesetzgeber oder die Praxis aufgeworfen. Der eingespielt offene Diskurs von Wissenschaft und richterlicher Praxis bewahrt die einen vor grauer Theorie, die anderen vor unverbundenen Entscheidungen des Einzelfalls.

Die Mitglieder des Bundesgerichtshofs haben sich diesem Diskurs für die jeweiligen Senatsmaterien von jeher gerne gestellt und diesen wo möglich maßgeblich mitgestaltet. Dieses allen Beteiligten dienende Element höchster Fachlichkeit repräsentiert der Jubilar der vorliegenden Festschrift in vorbildlicher Weise. Langjährig in den wissenschaftlichen Dialog als Richter, Autor, Kommentator, Lehrender und Vortragender eingebunden, vertritt er vor allem die Kernmaterie „seines“ IX. Zivilsenates in paradigmatischer Weise. 
Godehard Kayser, in Berlin geboren, wurde am 3. Dezember 1981 in den richterlichen Dienst des Landes Nordrhein-Westfalen aufgenommen, nachdem er die erste und zweite juristische Staatsprüfung in Hamm und Düsseldorf sehr erfolgreich abgelegt hatte. Den jungen Richter erwarteten die üblichen Stationen an verschiedenen Amts- und Landgerichten sowie dem Oberlandesgericht Hamm, die ihn als Proberichter und Richter am Landgericht 10jährige Erfahrungen in vielen Bereichen des Zivilrechts sowie der Freiwilligen Gerichtsbarkeit sammeln ließen. Es sind Jahre, die neben der Fachlichkeit auch der Entwicklung einer Richterpersönlichkeit zugeordnet werden können, die sich als überaus leistungsstark, verantwortungsbewusst und mit allen Anlagen wissenschaftlicher Befähigung empfahl. Zugleich wurde auch schon in diesen frühen Berufsjahren ein Faible für die Ausbildung des juristischen Nachwuchses durch Nebentätigkeiten als Korrekturassistent an der Westfälischen Wilhelms-Universität, in Referendararbeitsgemeinschaften, als Prüfer und später als Mitglied des Justizprüfungsamtes sowie Lehrbeauftragter an der Wilhelms-Universität ersichtlich.

Entscheidenden Anteil an den - aus der Rückschau so stimmig und konsequent verfolgt erscheinenden - beruflichen Wegen dürfte eine erste $\mathrm{Ab}$ ordnung an den Bundesgerichtshof als junger Familienvater zur wissenschaftlichen Mitarbeit in den IX. Zivilsenat gehabt haben. Ausgestattet mit der hier sehr erfolgreich erworbenen weiteren Kompetenz als wissenschaftlicher Mitarbeiter empfahl sich Godehard Kayser zur Abordnung auch an das Bundesverfassungsgericht, wo er Herrn Richter des Bundesverfassungsgerichts Winter einer ebenso erfahrenen wie prägenden Persönlichkeit u. a. bei bedeutenden Senatsentscheidungen zuarbeiten durfte. Ausgestattet nun auch mit dem Werkzeug des verfassungsrechtlichen Floretts diente Godehard Kayser weitere erfolgreiche Jahre dem Oberlandesgericht Hamm, u. a. in dem für das Konkurs-, Vergleichs-, Zwangsversteigerungsund Zwangsverwaltungsrecht zuständigen 15. Zivilsenat. Im Jahr der Jahrtausendwende wurde er auf Vorschlag des Justizministers des Landes Nordrhein-Westfalen zur Wahl als Richter am Bundesgerichtshof vorgeschlagen und bereits bei der unmittelbar darauffolgenden Wahl im Jahr 2001 zum Richter am Bundesgerichtshof gewählt, wo er seither dem IX. Zivilsenat mit den Schwerpunkten Insolvenzrecht, Zwangsvollstreckungsrecht, aber auch dem Recht der Rechtanwalts- und Steuerberaterhaftung zugewiesen ist. Mit einer Promotion zur „Lebensversicherung in der Insolvenz des Arbeitgebers - Zugleich ein Beitrag zur Erfüllungswabl und Erfüllungsablehnung“ sowie weiterer Lehrtätigkeit an „seiner“ Universität widmete 
Godehard Kayser seine wissenschaftlichen Leistungen nunmehr ganz dem Bereich des Insolvenzrechts, dem neben seiner Familie seine zweite große Leidenschaft gilt. Die Verleihung des Titels eines Honorarprofessors 2008 würdigte die jahrelangen Verdienste in Lehre und Forschung und manifestierte die Brücke, die zwischen praktischer Fragestellung und wissenschaftlicher Analyse zu schlagen ist. Mit seiner Ernennung zum Vorsitzenden Richter am Bundesgerichtshof und der Bestellung zum Vorsitzenden Richter des IX. Zivilsenats im Jahr 2010 konnte das vorbezeichnete überaus erfolgreiche Wirken auch dienstrechtlich nachvollzogen werden.

Godehard Kayser steht aber auch wie wenige andere für das in besonderer Weise verfassungsrechtlich geprägte anwaltliche Berufsrecht. Neben seiner Tätigkeit im IX. Zivilsenat hat Godehard Kayser auch beim Anwaltssenat des Bundesgerichtshofs seit vielen Jahren, ab dem 1. Januar 2012 zugleich als dessen stellvertretender Vorsitzender mit eigenständiger Zuständigkeit neben den von Gesetzes wegen zu Vorsitzenden dieses Senats berufenen Präsidenten des Bundesgerichtshofs, Herausragendes geleistet.

Mit einer wegweisenden Entscheidung des Bundesverfassungsgerichts vom 14. Juli 1987, der sog. „Bastilleentscheidung“ in Anlehnung an den Kampf um die Bastille am 14. Juli 1789, hatte das Bundesverfassungsgericht eine Doppelstrategie der Verantwortlichkeit einerseits des Gesetzgebers für grundlegende Abwägungen des Berufsrechts und andererseits die ausfüllende Verantwortung der satzungsgebenden Selbstverwaltungsorgane der Rechtsanwaltschaft festgelegt, die einer berufsrechtlichen Wesentlichkeitstheorie entspricht. Die Ablösung der seinerzeitigen Standesrichtlinien als verbindlichen Entscheidungsgrundlagen des anwaltlichen Berufsrechts zugunsten einer dem Sachlichkeitsgebot verpflichteten Berufsausübung in gesetzlichem Rahmen hat in der Folge eine Vielzahl grundlegender Entscheidungen der anwaltlichen Berufsgerichte und des Anwaltssenats beim Bundesgerichtshof ausgelöst.

Unter dem Vorsitz von Godehard Kayser sind ungezählte, nicht mehr hinweg zu denkende Entscheidungen zu Grundlagen des anwaltlichen Berufsrechts getroffen worden, darunter zuletzt auch solche zu der das anwaltliche Selbstverständnis revolutionierenden jungen Profession der Syndikusrechtsanwälte. Dabei hat sich Godehard Kayser einerseits als wertkonservativer Bewahrer der - besonderen Bindungen an die Belange der rechtssuchenden Allgemeinheit unterliegenden - traditionellen Anwaltschaft ebenso verpflichtet gesehen wie der moderaten Öffnung des Berufsstandes 
für neue Anforderungen und nicht zuletzt europarechtlichen Bindungen folgenden Erwägungen zur Liberalisierung des Berufsbildes.

Neben der Grundsätzliches klärenden und zugleich unmittelbar rechtsgestaltend wirkenden Rechtsprechung des Anwaltssenats selbst war und ist es Godehard Kayser dabei ein besonderes Anliegen und eine ganz besondere Freude, auch mit den anwaltlichen Beisitzern des Senats, regelmäßig besonders engagierten und fachlich ausgewiesenen Vertreterinnen und Vertretern ihres Fachs, den kollegialen Kontakt zu fördern und auszubauen. Bei einer diesem Anliegen dienenden Zusammenkunft aller richterlichen und anwaltlichen Mitglieder des Anwaltssenats gab Godehard Kayser der Autorin dieser Zeilen den entscheidenden Hinweis auf die Notwendigkeit der Bepflanzung einer Brachfläche auf dem Gelände des Bundegerichtshofs, der - wesenstypisch für diesen vorzüglichen Kollegen - sogleich mit einem konkreten Vorschlag versehen wurde. Seither ist mit der Bodendeckerrose Heidetraum neben allem Fachlichen auch ein bleibendes botanisches „Kayserdenkmal“ geschaffen, das in seiner blühenden Dimension auch sinnbildlich für einen in jeder Hinsicht Großen seiner Zunft steht. 